\title{
Rational Observers of Rational Systems
}

\author{
Jana Němcová and Mihály Petreczky and Jan H. van Schuppen
}

\begin{abstract}
Rational observers are to be constructed for rational systems while polynomial observers are to be constructed for polynomial systems. An observer synthesis procedure is formulated. First an output-based rational realization is synthesized for the considered rational system. Then a perturbation technique creates an observer. Finite algebraic observability of the rational system impies the existence of a output-based rational realization. Several examples of rational observers are provided including a polynomial system of which the statespace dimension of the polynomial observer is strictly higher than that of the corresponding system.
\end{abstract}

\section{INTRODUCTION}

The aim of this paper is to show how, for a rational system, a rational observer can be synthesized. The synthesis procedure is illustrated by several examples.

In control theory there is a need for observers of systems. Observers are used to produce estimates or predictions of values of the output of a system.

There is a large body of literature on observers of nonlinear systems. The reader is referred to the next section for a brief literature review.

The focus of this paper is on rational systems which arise in biochemical reaction systems, in physiological systems, and in engineering. These systems have been investigated by the authors in various papers, [1], [2], [3]. The scope of the investigation is restricted by imposing the condition that for a rational system one wants a rational observer while for a polynomial system one wants a polynomial observer. This is a self-imposed restriction but it makes sense considering the algebraic framework of rational systems.

A procedure for observer synthesis is proposed. The procedure consists of several steps of which the first one is the construction of an output-based realization followed later on by an output injection step. That observers are basically output-based realizations of the system generating the output is due to R.E. Kalman, [4], and to the subsequent research on stochastic realization.

In general a rational or a polynomial observer may have a higher state-space dimension than the dimension of the system. Example VII.1 of a polynomial system provides a polynomial observer of a strictly higher state-space dimension than the system.

This work was partially supported by the GAČR project 13-16764P. J. Němcová is with the Department of Mathematics, University of Chemistry and Technology, Technická 5, 16628 Prague 6, Czech republic Jana. Nemcova@vscht.cz

M. Petreczky is with the Ecole Centrale Lille, 59651 Villeneuve d'Asq, Lille, France mihaly.petreczky@ec-lille.fr

J.H. van Schuppen is with the company Van Schuppen Control Research, Gouden Leeuw 143, 1103 KB Amsterdam, The Netherlands jan.h.van.schuppen@xs4all.nl
The outline of the paper follows. The next section provides a problem description and motivation. Section [III defines rational systems and shows how to check their observability. Section IV provides the procedure for observer synthesis. The theory on which the procedure is based is provided in Section $\mathrm{V}$. The performance of the observer is discussed in SectionVI, Section VII] shows several examples of observers.

\section{PROBLEM FORMULATION}

The motivations for the synthesis of an observer of a system are primarily: (1) the interest in estimation of the state of the observer for example if the state is the concentration of a chemical species or if it is a concentration of a physiological model of a human being; (2) the starting point for prediction of a time series for example prediction of traffic flow in a road network, [5], or prediction of produced photo-voltaic power of solar panels; (3) control based on partial observations, [6]; and (4) the use of observers in system identification, [7].

There is an extensive literature on observers of control systems. The foundation is the publication of the Kalman filter, [8], followed by the papers of D. Luenberger on an observer of a linear system, [9], [10]. By now there is an extensive literature on observers of several classes of nonlinear systems, [11]. Recent books exclusively on observers include [12], [13] while major papers include [14], [15], [16].

What is the definition of an observer? Several definitions for an observer have been proposed. In this paper an observer will be based on an output-based realization of a nonlinear system, meaning a realization of which the state is a function of the output and of the output's derivatives. From such a realization one can directly construct the observer. The view point of an observer being based on an output-based realization of the system, is inspired by the publications of Kalman on the stochastic realization theory of Kalman filters, see [4].

A restriction is imposed on the algebraic form of the observers. For a polynomial system the search is restricted to a polynomial observer and for a rational system the search is restricted to a rational observer. It should be clear that this is a self-imposed restriction, there may exists observers in a wider class of systems for example in the class of Nash systems defined by the authors in [3]. A consequence of this restriction on the algebraic form of the observer is that the observer may have a higher dimension than the corresponding system, see Example VII.1 below.

The performance of the observer is investigated. The initial condition of the observer is related to the output function 
and its derivatives which derivatives are often not directly available. Therefore, there is a convergence issue in case the observer is started in an initial state which is different from that of an output-based realization. Stability analysis of observers is difficult and one has to prove that the prediction of the observer for the output converges to the observed output. This performance criterion is discussed but not completely treated in this short conference paper.

Problem II.1 Observer Synthesis. Consider a rational system without inputs,

$$
\begin{aligned}
\frac{d x(t)}{d t} & =f(x(t)), \quad x(0)=x_{0} \in \mathbb{R}^{n}, \\
y(t) & =h(x(t)) .
\end{aligned}
$$

Synthesize an observer of the form,

$$
\begin{aligned}
\frac{d x_{o}(t)}{d t} & =f_{o}\left(x_{o}(t), y(t)\right), \quad x_{o}(0)=x_{o, 0} \in \mathbb{R}^{n_{o}}, \\
y_{o}(t) & =h_{o}\left(x_{o}(t)\right), \text { such that, } \\
0 & =\lim _{s \rightarrow \infty}\left[y(s)-y_{o}(s)\right], \text { and, }
\end{aligned}
$$

- if the system is a rational system ( $f$ and $h$ rational maps), then the observer is a rational system ( $f_{o}$ and $h_{o}$ are rational maps); and

- if the system is a polynomial system ( $f$ and $h$ are polynomial maps), then the observer is a polynomial system, ( $f_{o}$ and $h_{o}$ are polynomial maps).

Realization theory of discrete-time polynomial systems was formulated by E.D. Sontag in his Ph.D. thesis, [17], and generalized by $\mathrm{Z}$. Bartosiewicz to continuous-time polynomial systems, [18]. Realization theory of continuous-time nonlinear and rational systems was initiated in [19], [20] and further developed by by J. Němcová, [1], [2].

\section{RATIONAL SYSTEMS}

The concepts of rational systems and their observability recalled in this section are adopted from [18], [1], [2]. To state the proper definitions, let us first provide a short overview of necessary terms from commutative algebra and algebraic geometry. For more details see e.g. [21], [22], [23], [24].

By $\mathbb{R}\left[X_{1}, \ldots, X_{n}\right]$ we denote the algebra of polynomials in $n$ variables with coefficients in the real numbers. A subset $X \subseteq \mathbb{R}^{n}$ is called a variety if it is a set of points of $\mathbb{R}^{n}$ which satisfy finitely many polynomial equalities. We say it is irreducible if it cannot be written as a union of two disjoint varieties. Let $I(X)$ denote the ideal of polynomials of $\mathbb{R}\left[X_{1}, \ldots, X_{n}\right]$ vanishing on $X$. Then the elements of $\mathbb{R}\left[X_{1}, \ldots, X_{n}\right] / I(X)$ are referred to as polynomials on $X$. The ring of all such polynomials is denoted by $A_{X}$. Since $X$ is irreducible and thus $A_{X}$ is an integral domain, one can define $Q_{X}$, the field of rational functions on $X$, as a field of fractions of $A_{X}$.
Definition III.1 By a rational system $\Sigma$ (without inputs) we refer to a control system as understood in system theory, [25], with the notation,

$$
\begin{aligned}
\Sigma & =\left(X, Y, f, h, x_{0}\right), \\
\frac{d x(t)}{d t} & =f(x(t)), x(0)=x_{0} \in X, \\
y(t) & =h(x(t)),
\end{aligned}
$$

where the state-space $X$ is an irreducible variety in $\mathbb{R}^{n}, Y=$ $\mathbb{R}^{m_{y}}$ with $m_{y} \in \mathbb{Z}_{+}$, and the components of $f: X \rightarrow \mathbb{R}^{n}$ and $h: X \rightarrow \mathbb{R}^{m_{y}}$ are rational maps on $X$ defined at $x_{0}$.

Polynomial systems are defined analogically with the components of $f$ and $h$ being polynomial maps on $X$.

Example III.2 Rational systems are widely used, among others, for mathematical description of biological phenomena. One such example is the following rational system $\Sigma$ which describes an enzyme catalyzed change of a substrate to a product. The structure of $\Sigma$ is derived by considering the corresponding single reversible reaction to be modeled by Michaelis-Menten kinetics. Let $x_{1}$ denote the substrate concentration and let $x_{2}$ denote the product concentration, then $\Sigma$ is described by the equations,

$$
\begin{aligned}
\frac{d x_{1}(t)}{d t} & =-a x_{1}(t)+\frac{c x_{1}(t)+b x_{1}^{2}(t)}{x_{1}(t)+d}, x_{1}(0)=1, \\
\frac{d x_{2}(t)}{d t} & =\frac{e x_{1}(t)}{x_{1}(t)+d}, x_{2}(0)=1, \\
y(t) & =x_{2}(t),
\end{aligned}
$$

where the considered state-space $X$ equals $\mathbb{R}^{2}$, the concentration $x_{2}$ of the product is assumed to be observed, the initial conditions are chosen to be positive, and constant parameters $a, b, c, d, e$ have specific biological meaning. Let us, for simplicity, assume $a=b=c=e=1$ and $d=2$.

In the remainder of the paper the concept of observability of a rational system is needed which is thus borrowed from [1], [2].

Definition III.3 Consider a rational system $\Sigma=$ $\left(X, Y, f, h, x_{0}\right)$. Define the observation algebra $A_{\text {obs }}(\Sigma)$ of $\Sigma$ as the algebra over the real numbers generated by the components of $h$ and closed with respect to Lie derivatives along the vector field $f$. Thus,

$$
A_{o b s}(\Sigma)=\mathbb{R}\left[\left\{L_{f}^{k} h_{i} \mid i=1, \ldots, m, k=0,1, \ldots\right\}\right],
$$

where $L_{f}^{0} h_{i}=h_{i}, L_{f}^{1} h_{i}=L_{f} h_{i}=\sum_{j=1}^{n} f_{j}(x) \frac{\partial}{\partial x_{j}} h_{i}$, and $L_{f}^{k} h_{i}=L_{f}\left(L_{f}^{k-1} h_{i}\right)$ for $k=2,3 \ldots, i=1, \ldots, m$. Because $X$ was an irreducible variety, one can define the observation field $Q_{o b s}(\Sigma)$ of $\Sigma$ as the field of fractions of $A_{o b s}(\Sigma)$, i.e.

$$
Q_{o b s}(\Sigma)=\left\{p / q \mid p, q \in A_{o b s}(\Sigma), q \neq 0\right\} .
$$

The rational system $\Sigma$ is called algebraically observable if its observation field equals the field of all rational functions on the state-space, $Q_{X}=Q_{o b s}(\Sigma)$. 
The polynomial system $\Sigma$ is called algebraically observable if its observation algebra equals the algebra of all polynomials on the state-space, $A_{X}=A_{o b s}(\Sigma)$.

Example III.4 Consider the rational system defined in Example III.2 Let us refer to it as to $\Sigma=\left(X, Y, f, h, x_{0}\right)$. Then,

$$
L_{f} h\left(x_{1}, x_{2}\right)=\frac{-x_{1}}{x_{1}+2} \frac{\partial h\left(x_{1}, x_{2}\right)}{\partial x_{1}}+\frac{x_{1}}{x_{1}+2} \frac{\partial h\left(x_{1}, x_{2}\right)}{\partial x_{2}}
$$

and $h\left(x_{1}, x_{2}\right)=x_{2}$. Let us compute few elements of $Q_{o b s}(\Sigma)$ :

$$
\begin{aligned}
h & =x_{2} \in Q_{o b s}(\Sigma) \\
L_{f}\left(x_{2}\right) & =\frac{x_{1}}{x_{1}+2} \in Q_{o b s}(\Sigma), \\
L_{f}\left(\frac{x_{1}}{x_{1}+2}\right) & =\frac{-2 x_{1}}{\left(x_{1}+2\right)^{3}} \in Q_{o b s}(\Sigma) .
\end{aligned}
$$

To obtain further elements one can keep on computing Lie derivatives of known elements, multiply and divide the elements and multiply them by real numbers. For example, $\frac{-2 x_{1}}{\left(x_{1}+2\right)^{3}} \frac{-1}{2}=\frac{x_{1}}{\left(x_{1}+2\right)^{3}} \in Q_{o b s}(\Sigma)$ and $\frac{x_{1}}{\left(x_{1}+2\right)} \frac{\left(x_{1}+2\right)^{3}}{x_{1}}=$ $\left(x_{1}+2\right)^{2} \in Q_{o b s}(\Sigma)$. Then, $L_{f}\left(\left(x_{1}+2\right)^{2}\right)=-2 x_{1}$ and consequently $x_{1}$ belong to $Q_{o b s}(\Sigma)$. Because $x_{1}, x_{2} \in$ $Q_{o b s}(\Sigma)$, it follows that $Q_{o b s}(\Sigma)=\mathbb{R}\left(x_{1}, x_{2}\right)=Q_{X}$ which implies $\Sigma$ is algebraically observable.

\section{OBSERVER SYNTHESIS}

The observer synthesis procedure is stated below and is complemented in the next section by theory of the various steps. Below either a rational or a polynomial system is considered. A polynomial system is taken along in the exposition to illustrate the various steps.

\section{Procedure IV.1 Observer Synthesis Procedure}

1) Construct a state-space transformation $s: X \rightarrow \hat{X}$.

2) Prove the existence of an inverse function $s^{-1}$ of $s$ of the state-space transformation in the subclass of rational or of polynomial functions depending on the case.

3) Derive an output-based realization.

4) Derive the observer.

5) Choose an observer gain to meet performance objectives, including stability.

Example IV.2 Polynomial system. Consider the observable polynomial system for which an observer is wanted.

$$
\begin{aligned}
\frac{d x(t)}{d t}= & \left(\begin{array}{l}
-a_{11} x_{1}(t)^{3}+a_{12} x_{2}(t) \\
-a_{22} x_{2}(t)
\end{array}\right), x(0)=x_{0}, \\
y(t)= & x_{1}(t)=C x(t)=\left(\begin{array}{cc}
1 & 0
\end{array}\right) x(t), \\
& a_{11}, a_{22} \in(0, \infty), a_{12} \in \mathbb{R}, a_{12} \neq 0 .
\end{aligned}
$$

Procedure IV.3 Step 1. Construction of a state-space transformation. Define,

$$
\begin{aligned}
s_{1}(x)= & h(x), s_{1}: X \rightarrow \mathbb{R}^{m_{y}}, \quad y(t)=h(x(t)), \\
\frac{d y(t)}{d t}= & \frac{d h(x(t))}{d t}=\left.\frac{\partial h(x)}{\partial x}\right|_{x=x(t)} \frac{d x(t)}{d t} \\
= & \left.\frac{\partial h(x)}{\partial x} f(x)\right|_{x=x(t)}=s_{2}(x(t)), \\
s_{2}(x)= & \frac{\partial s_{1}(x)}{\partial x} f(x), \quad s_{2}: X \rightarrow \mathbb{R}^{m_{y}}, \\
s_{k+1}(x)= & \frac{\partial s_{k}(x)}{\partial x} f(x), k \in \mathbb{Z}_{+}, \\
& m_{o} \in \mathbb{Z}_{+}, n_{o}=m_{o} \times m_{y}, \\
s_{1}(x)= & \left(\begin{array}{c}
s_{1}(x) \\
s_{2}(x) \\
\vdots \\
s_{m_{o}}(x)
\end{array}\right), \quad s: X=\mathbb{R}^{n} \rightarrow \mathbb{R}^{n_{o}} .
\end{aligned}
$$

The choice of the rational observability index $m_{o} \in \mathbb{Z}_{+}$is best made in Step 2 based on the existence of the inverse of the state-space transformation $s$ in a specified set.

Example IV.4 Polynomial system. Example IV.2 is continued. The state-space transformation function is calculated.

$$
\begin{aligned}
s_{1}(x) & =h(x)=x_{1}, \quad s_{2}(x)=-a_{11} x_{1}^{3}+a_{12} x_{2} \\
s(x) & =\left(s_{1}(x), s_{2}(x)\right)^{T}
\end{aligned}
$$

Procedure IV.5 Step 2. Prove the existence and construct a rational inverse or a polynomial inverse of the state-space transformation,

$$
\begin{aligned}
\hat{x}(t)= & \left(y(t) \quad \frac{d y(t)}{d t} \quad \ldots \quad \frac{d^{m_{o}-1} y(t)}{d t^{m_{o}-1}}\right)^{T}, \\
\hat{x}: T \rightarrow \mathbb{R}^{n_{o}} & \\
\hat{x}(t)= & s(x(t)), s \in Q_{o b s}(\Sigma)^{n_{o}} .
\end{aligned}
$$

Construct an inverse function $s^{-1}$ of the state-space transformation $s$ such that if $s$ is a rational, then $s^{-1}$ will be a rational while if $s$ is a polynomial, then $s^{-1}$ will be a polynomial.

It is proven in the next section that observability of the rational or polynomial system implies the existence of the inverse in the required class of algebraic objects.

Example IV.6 Polynomial system. Example IV.4 is continued. The state-space transformation $s$ admits a polynomial inverse,

$$
\begin{aligned}
\hat{x}_{1} & =s_{1}(x)=x_{1}, x_{1}=\hat{x}_{1}, \\
\hat{x}_{2} & =s_{2}(x)=-a_{11} x_{1}^{3}+a_{12} x_{2}, \\
s^{-1}(\hat{x}) & =\left(\begin{array}{l}
x_{1} \\
x_{2}
\end{array}\right)=\left(\begin{array}{l}
\hat{x}_{1} \\
\frac{a_{11}}{a_{12}} \hat{x}_{1}^{3}+\frac{1}{a_{12}} \hat{x}_{2}
\end{array}\right) .
\end{aligned}
$$


Procedure IV.7 Step 3. Derivation of the output-based realization. Next the output-based realization can be calculated,

$$
\begin{aligned}
x(t) & =s^{-1}(\hat{x}(t)), \\
\frac{d \hat{x}(t)}{d t} & =f_{o r}(\hat{x}(t)), \\
y(t) & =h_{o r}(\hat{x}(t))=C_{o} \hat{x}(t)=\hat{x}_{1: m_{y}}(t), \\
f_{o r}(\hat{x}) & =\left.\frac{\partial s(x)}{\partial x} f(x)\right|_{x=s^{-1}(\hat{x})}, \\
C_{o} & =\left(\begin{array}{llll}
I_{m_{y}} & 0 & \ldots & 0
\end{array}\right) .
\end{aligned}
$$

Definition IV.8 Special case of single output. Below attention is restricted to a system with a one-dimensional output, hence $m_{y}=1$. Then $n_{o}=m_{o}$. Note that then,

$$
\begin{aligned}
& \hat{x}(t)=\left(\begin{array}{llll}
y(t) & \frac{d y(t)}{d t} & \ldots & \frac{d^{n_{o}-1} y(t)}{d t^{n_{o}-1}}
\end{array}\right)^{T}, \\
& \frac{d}{d t} \hat{x}_{i}(t)=\frac{d}{d t} \frac{d^{i-1} y(t)}{d t^{i-1}}=\frac{d^{i} y(t)}{d t^{i}}=\hat{x}_{i+1}(t), \\
& \frac{d}{d t} \hat{x}_{n_{o}}(t)=\frac{d}{d t} \frac{d^{n_{o}-1} y(t)}{d t^{n_{o}-1}}=\frac{d^{n_{o}} y(t)}{d t^{n_{o}}}=s_{n_{o}+1}(x(t)) . \\
& \frac{d \hat{x}(t)}{d t}=f_{o r}(\hat{x}(t))=A_{o} \hat{x}(t)+b_{o}(\hat{x}(t)), \\
& A_{o}=\left(\begin{array}{ccccc}
0 & 1 & 0 & \ldots & 0 \\
0 & 0 & 1 & \ldots & 0 \\
\vdots & & \ddots & & \vdots \\
0 & 0 & 0 & \ldots & 1 \\
0 & 0 & 0 & 0 & 0
\end{array}\right), \\
& b_{o}(\hat{x})=0, \quad \text { except that, } \\
& b_{o, n_{o}}(\hat{x})=s_{n_{o}+1}\left(s^{-1}(\hat{x})\right)=\left.\frac{\partial s_{n_{o}}(x)}{\partial x} f(x)\right|_{x=s^{-1}(\hat{x})} .
\end{aligned}
$$

The same structure holds in the multi-output case, with $m_{y}>1$ though then the matrix $A_{o}$ and the vector $b_{o}$ have multivariable components.

Example IV.9 Polynomial system. Example IV.6 is continued. The output-based realization is,

$$
\begin{aligned}
\frac{d \hat{x}(t)}{d t} & =\left(\begin{array}{l}
\hat{x}_{2}(t) \\
\hat{f}_{o r, 2}(\hat{x}(t))
\end{array}\right), \hat{x}(0)=s(x(0)), \\
\hat{f}_{o r, 2}(\hat{x}) & =-a_{11} a_{22} \hat{x}_{1}^{3}-3 a_{11} \hat{x}_{1}^{2} \hat{x}_{2}-a_{22} \hat{x}_{2}, \\
y(t) & =C_{o r} \hat{x}(t)=\hat{x}_{1}(t) .
\end{aligned}
$$

In an output-based realization, the output is a component of the state vector of the realization. What is needed is that the output of the system becomes an input of the observer. This can be achieved by replacing the first component of the output-based realization by an abstract variable and by injecting the output into the first component. Due to the fact that in the output-based realization the output function is linear, the injection of the output in the observer is with the linear function $y(t)-C_{o} x_{o}(t)$.
Procedure IV.10 Step 4. Derivation of the observer by output injection. Define the observer as the system,

$$
\begin{aligned}
& \frac{d x_{o}(t)}{d t}=A_{o} x_{o}(t)+b_{o}\left(x_{o}(t)\right)+ \\
& +\left[k_{o}\left(x_{o}(t)\right)+K\right]\left[y(t)-C_{o} x_{o}(t)\right], \\
& x_{o}\left(t_{0}\right)=x_{o, 0} \in \mathbb{R}^{n}, K \in \mathbb{R}^{n_{0} \times m_{y}} \text {, } \\
& k_{o}\left(x_{o}\right)=\left(\begin{array}{llll}
0 & 0 & \ldots & k_{o, m_{o}}\left(x_{o}\right)
\end{array}\right)^{T} \in \mathbb{R}^{n_{o} \times m_{y}}, \\
& k_{o, m_{o}}\left(x_{o}\right)=\frac{\partial b_{o, m_{o}}\left(x_{o}\right)}{\partial x_{o, 1}} \in \mathbb{R}^{m_{y} \times m_{y}} \text {. }
\end{aligned}
$$

An explanation of the derivation of the observer follows for the case $m_{y}=1$. Apply a Taylor expansion to the outputbased realization, and retain only the first-order term, [26].

$$
\begin{aligned}
& \frac{d \hat{x}(t)}{d t}=f_{\text {or }}(\hat{x}(t)), \quad \hat{x}(0)=\hat{x}_{0} \in \mathbb{R}^{n_{o}}, \\
& y(t)=h_{o r}(\hat{x}(t)) \text {, } \\
& \hat{x}(t)=\left(\begin{array}{lllll}
\hat{x}_{1}(t) & \hat{x}_{2}(t) & \hat{x}_{3}(t) & \ldots & \hat{x}_{n_{o}}(t)
\end{array}\right)^{T}, \\
& \bar{x}(t)=\left(\begin{array}{lllll}
y(t) & \hat{x}_{2}(t) & \hat{x}_{3}(t) & \ldots & \hat{x}_{n_{o}}(t)
\end{array}\right)^{T}, \\
& f_{\text {or }}(\hat{x}(t)) \\
& =f_{o r}(\bar{x}(t))+\left[f_{\text {or }}(\hat{x}(t))-f_{o r}(\bar{x}(t))\right] \text {, } \\
& \approx f_{o r}(\bar{x}(t))+\left.\frac{\partial f_{o r}(x)}{\partial x}\right|_{x=\bar{x}(t)}[\hat{x}(t)-\bar{x}(t)] \\
& \left.=f_{o r}(\bar{x}(t))+\left.\frac{\partial f_{o r}(x)}{\partial x_{1}}\right|_{x=\bar{x}(t)}\left[y(t)-C_{o} \bar{x}(t)\right)\right], \\
& \frac{d x_{o}(t)}{d t}=f_{o}\left(x_{o}(t)\right)+\left[k_{o}\left(x_{o}(t)\right)+K\right]\left[y(t)-C_{o} x_{o}(t)\right] \text {. }
\end{aligned}
$$

The choice for a gain matrix $K \in \mathbb{R}^{n_{o} \times m_{y}}$ made above is expected to guarantee local stability for specific values. Further research is required on the global asymptotic stability of the performance system, see Section VI, to allow the formulation of nonlinear gain functions.

Example IV.11 Polynomial system. Example IV.9 is continued.

$$
\begin{aligned}
\frac{d x_{o}(t)}{d t}= & \left(\begin{array}{l}
x_{o, 2}(t) \\
f_{o, 2}\left(x_{o}(t), y(t)\right)
\end{array}\right)+ \\
& +\left[k_{o}\left(x_{o}(t)\right)+K\right]\left[y(t)-C x_{o}(t)\right] \\
= & \left(\begin{array}{l}
x_{o, 2}(t) \\
-a_{11} a_{22} x_{o, 1}^{3}-3 a_{11} x_{o, 1}^{2} x_{o, 2}-a_{22} x_{o, 2}
\end{array}\right) \\
& +\left(\begin{array}{l}
k_{1} \\
k_{2}-3 a_{11} a_{22} x_{o, 1}^{2}-6 a_{11} x_{o, 1} x_{o, 2}
\end{array}\right) \times \\
& \times\left[y(t)-C x_{o}(t)\right], \\
y_{o}(t)= & C x_{o}(t) .
\end{aligned}
$$

Procedure IV.12 Step 5. Choose the oberver gain. Choose the gain matrix such that the performance system of Def. VI.1 meets the performance objective of stability and good transient response.

\section{THEORY OF OBSERVER SYNTHESIS}

This section provides concepts and results which show that the synthesis procedure of the previous section produces indeed a rational or a polynomial observer. 
Consider a rational system. Def. [II.3 defined first the observation algebra of a rational system as the algebra of rational functions generated by the infinite sequence of Lie derivatives of the system for the output components and subsequently the observation field as the field of fractions of this observation algebra. Below a corresponding concept is defined for a finite sequence of these Lie derivatives of output components. Note that the finite set of the zero-th upto the $m$ th Lie derivative of the components of the output function are precisely the family of $\left\{s_{i}, i \in \mathbb{Z}_{m+1}\right\}$ functions.

The exposition below was first written for the single output case, thus for $m_{y}=1$. It can be read also for the multi-output case, for $m_{y} \geq 2$, if the sequence of $s$ functions and their components are renumbered. For the rest of the section, renumber the functions, $s_{1,1}, s_{1,2}, \ldots, s_{1, m_{y}}, s_{2,1}, \ldots, s_{2, m_{y}}, s_{3,1}, \ldots$ as $s_{1}, s_{2}, \ldots$, i.e. $s_{1}=s_{1,1}, s_{2}=s_{1,2}, \ldots, s_{m_{y}}=s_{1, m_{y}}, s_{m_{y}+1}=s_{2,1}$, ...

Definition V.1 Finite algebraic observability of a rational and of a polynomial system.

(a) Consider a rational system $\Sigma=\left(X, Y, f, h, x_{0}\right)$ and let the functions $\left\{s_{i}: \mathbb{R}^{n} \rightarrow \mathbb{R}, i \in \mathbb{Z}_{+}\right\}$be as constructed in Procedure IV.3 Define the $m$-th observation algebra of rational functions and the associated $m$-th observation field for all $m \in \mathbb{Z}_{+}$as respectively,

$$
\begin{aligned}
& A_{o b s, m}(\Sigma)=\mathbb{R}\left[\left\{s_{1}, s_{2}, \ldots, s_{m}\right\}\right], \\
& Q_{o b s, m}(\Sigma)=\left\{p / q \mid p, q \in A_{o b s, m}(\Sigma), q \neq 0\right\} .
\end{aligned}
$$

Call the rational system finitely algebraically observable if there exists an integer $m \in \mathbb{Z}_{+}$such that $Q_{o b s, m}(\Sigma)=\mathbb{R}(x)$.

Call the least-integer $m \in \mathbb{Z}_{+}$such that $Q_{\text {obs }, m}(\Sigma)=$ $\mathbb{R}(x)$, the rational observability index of the rational system, denote it by $m_{o} \in \mathbb{Z}_{+}$, and let $n_{o}=m_{o} * m_{y}$ (with disregard of renumbering).

(b) Consider a polynomial system. Define correspondingly for this polynomial system the $m$-th observation algebra of polynomial functions, finite algebraic observability, and the polynomial observability index.

The concept of a rational observability index and its polynomial analogue are analogous to those for linear systems, see [27, p. 356-357]. Less useful for observers because of robustness reasons seems the concept of rational Kronecker indices, see [28] for those of linear systems. Each such index for a component of the output function is defined as the least integer for which the rational observation field of that component stops increasing forever. The value of these indices depends on the ordering of the output components.

It follows from [1, Prop. 5.7] that if a rational system is algebraically observable then its observation field is finitely generated hence the rational system is finitely algebraically observable. In the case of a polynomial system it is not known whether algebraic observability implies that this polynomial system is finitely algebraically observable. Therefore the finite algebraic observability will be assumed.
Finite algebraic observability of a rational or of a polynomial system does not imply the existence of an inverse of $s$ with $m \geq\left\lceil n / m_{y}\right\rceil$. Example VII.1 of a polynomial system shows that the polynomial observability index can be strictly higher than the state-space dimension of the system, or, equivalently, with $m_{y}=1, n_{o}>n$.

Proposition V.2 Algebraic characterization of the existence of a rational or of a polynomial inverse of the statespace transformation. Consider a polynomial system $\Sigma=$ ( $\left.X, Y, f, h, x_{0}\right)$, the functions $\left\{s_{i}, i \in \mathbb{Z}_{+}\right\}$, and the family of observation algebras $\left\{A_{\text {obs }, m}(\Sigma), m \in \mathbb{Z}_{+}\right\}$.

$$
\begin{aligned}
& \exists m \in \mathbb{Z}_{+} \exists r_{1}, r_{2}, \ldots, r_{n} \in \mathbb{R}\left[S_{1}, \ldots, S_{m}\right], \\
& \text { and let } s=\left(s_{1}, \ldots, s_{m}\right), \\
& \text { such that, } x_{i}=r_{i}(s(x)), \forall i \in \mathbb{Z}_{n} ; \\
& \text { hence, } x=r(s(x)) \in A_{\text {obs }, m}(\Sigma)^{n} \text { and } s^{-1}=r ; \\
& \text { if and only if, } \\
& \exists m \in \mathbb{Z}_{+} \text {such that } A_{\text {obs }, m}(\Sigma)=\mathbb{R}[x] .
\end{aligned}
$$

Note that if there exists an $r$ as above, then $s$ is invertible with the polynomial inverse $s^{-1}=r$.

A corresponding result holds for the existence of a rational inverse $s^{-1}$ of $s$ in terms of the observation field $Q_{o b s, m}(\Sigma)$.

Proof. $\Leftarrow$ Because $\mathbb{R}[x] \subseteq A_{o b s, m}(\Sigma)$, for any $i \in \mathbb{Z}_{n}$ there exists a polynomial $r_{i}(s(x)) \in \mathbb{R}\left[x_{1}, \ldots, x_{n}\right]$ such that $x_{i}=$ $r_{i}(s(x))$. Define $r=\left(r_{1}, \ldots, r_{n}\right)$. Then,

$$
x=r(s(x))=\left(\begin{array}{l}
r_{1}(s(x)) \\
\vdots \\
r_{n}(s(x))
\end{array}\right) \in\left(A_{o b s, m}(\Sigma)\right)^{n} .
$$

From this follows that $s^{-1}=r \in \mathbb{R}\left[S_{1}, \ldots, S_{m}\right]$ and thus that the inverse $s^{-1}$ of $s$ exists and that it has polynomial components.

$\Rightarrow$ If an inverse function $r$ in the indicated set exists then $x_{i}=r_{i}(s(x)) \in A_{o b s, m}(\Sigma)$ for all $i \in \mathbb{Z}_{n}$. Thus $\mathbb{R}[x] \subseteq$ $A_{o b s, m}(\Sigma) \subseteq \mathbb{R}[x]$ where the last inclusion relation is by definition of $A_{o b s, m}(\Sigma)$. Hence equality holds.

Theorem V.3 Existence of an output-based realization. Consider a rational or a polynomial system. If the system is finitely algebraically observable then there exists an outputbased realization which is rational or polynomial respectively. Thus, $\Sigma_{o r}=\left(X_{o r}, Y, f_{o r}, h_{o r}, x_{o r, 0}\right)$, as specified in Procedure IV.7 is a rational or a polynomial system respectively.

Proof. The case of a rational system is considered, the case of a polynomial system is similar. The definition of the state vector $\hat{x}$ as a function of the output $y$ and several of its derivatives makes clear that the realization is output based. Note that finite algebraic observability of the system implies that there exists a finite number $m_{o} \in \mathbb{Z}_{+}$such that $Q_{o b s, m_{o}}(\Sigma)=\mathbb{R}(x)$. From Proposition $\mathbf{V . 2}$ then follows that 
the function $s: \mathbb{R}^{n} \rightarrow \mathbb{R}^{n_{o}}$ has an inverse function $s^{-1}$ which is also rational. From the formulas of Procedure IV.7 Step 3, then follows that the functions $\left(f_{\text {or }}, h_{\text {or }}\right)$ are rational. In more detail, the inverse function $s^{-1}$ is a rational function, $f_{\text {or }}(\hat{x})=\left.(\partial s(x) / \partial x) f(x)\right|_{x=s^{-1}(\hat{x})}$, and the class of rational functions is closed with respect to substitution, thus $f_{\text {or }}$ is a rational function. Hence the output-based realization is a rational system.

The existence of a polynomial inverse of a polynomial map has been characterized for which purpose we quote the following results from [29].

Let $k$ be an arbitrary field of characteristic zero (note that for our problem $k=\mathbb{R}$ meets this condition). A necessary condition for a polynomial map $F \in k\left[X_{1}, \ldots, X_{n}\right]$ to be invertible is that $F$ satisfies the Jacobi condition, that $\operatorname{det}\left(J_{F}\right) \in k^{*}$, where $k^{*}$ denotes the units of $k$. In general, the Jacobi condition is not sufficient. In case $n=1$, one can prove the sufficiency. For $n \geq 2$ the problem whether $F \in$ $\mathbb{R}\left[X_{1}, \ldots, X_{n}\right]$ satisfying the Jacobi condition is invertible is known as the Jacobi conjecture.

By adding additional assumptions on polynomial maps satisfying the Jacobi condition, one can obtain sufficient conditions of the following form.

Theorem V.4 [29, Th. 2.2.16, p. 53]. Let $F \in$ $\mathbb{R}\left[X_{1}, \ldots, X_{n}\right]$ be such that $\operatorname{det}\left(J_{F}\right) \in k^{*}$. If $k[X]$ is integral over $k[F]$ or if the field extension of $k(F) \subset k(X)$ is Galois, then $F$ is invertible.

For polynomial maps $F \in \mathbb{R}\left[X_{1}, \ldots, X_{n}\right]$ with $k$ being an arbitrary field, A. van den Essen derived a criterion based on the theory of Gröbner bases which not only decides whether $F$ is invertible but also provides a procedure to calculate the inverse if it exists. For the respective theorem (more general than quoted here), see [29, Theorem 3.2.1, p.64] or [30].

Note that for $n=1$ the invertible polynomials $F \in k[X]$ are only the affine ones, for example $F(x)=a x+b$. For $n=2$ every invertible polynomials $F: k^{2} \rightarrow k^{2}$ is tame, meaning that it can be written as a finite composition of elementary maps $\left(X_{1}+a, X_{2}\right)$ and $\left(X_{1}, X_{2}+b\right)$. For $n=3$ it is an open problem to determine whether every invertible polynomial $F \in k\left[X_{1}, \ldots, X_{n}\right]$ is tame. The general belief is that they are not.

\section{PERFORMANCE ISSUES}

The performance objectives of observer synthesis are: (1) asymptotic stability of the difference of the output of the system and the output of the observer; and (2) a good transient response in reaction to realistic initial conditions of the observer. In control theory it is known that it is best for the functioning of the observer if the convergence of the predicted output of the observer to the observed output is slightly faster than the dynamics of the system. It is well known from other subareas of control theory that for the analysis of the performance of an observer one has to consider the performance system with as state $\left(x, x_{o}\right)$.
The approach to investigate the global asymptotic stability of the observer includes the steps: (1) Investigate local stability at zero error. (2) Approximate the domain of attraction by analysis and simulation. See [31] for a procedure to approximate the domain of attraction by rational Lyapunov functions.

Definition VI.1 Define the performance system of the $o b$ server as the control system,

$$
\begin{aligned}
x_{e}(t) & =\left(\begin{array}{c}
x(t) \\
x_{o}(t)
\end{array}\right), x_{e}(0)=\left(\begin{array}{c}
x_{0} \\
x_{o}, 0
\end{array}\right), \\
\frac{d x_{e}(t)}{d t} & =f_{e}\left(x_{e}(t)\right)=\left(\begin{array}{c}
f(x(t)) \\
f_{o}\left(x_{o}(t), C x(t)\right)
\end{array}\right), \\
e_{y}(t) & =y(t)-h_{o}\left(x_{o}(t)\right)=h(x(t))-h_{o}\left(x_{o}(t)\right) \\
& =h_{e}\left(x_{e}(t)\right) .
\end{aligned}
$$

Note that the dimensions of the vectors $x$ and $x_{o}$ are in general different hence it is mathematically not possible to subtract these vectors.

Problem VI.2 Can an observer be synthesized such that for any initial condition $x_{e}(0)$ one has that,

$$
\lim _{t \rightarrow \infty} e_{y}(t)=0 ?
$$

Equivalently, is the observable part of the performance system such that the output $e_{y}$ is asymptotically stable for all initial conditions of the performance system?

The problem above is briefly analyzed for linear systems.

Definition VI.3 Performance system of a linear observer.

$$
\begin{aligned}
& \frac{d x(t)}{d t}=A x(t), x(0)=x_{0}, y(t)=C x(t) . \\
& \frac{d x_{o}(t)}{d t}=A x_{o}(t)+K\left[y(t)-C x_{o}(t)\right], x_{o}(0)=x_{o, 0} . \\
& x_{e}(t)=\left(\begin{array}{ll}
x(t) & x_{o}(t)
\end{array}\right)^{T} \text {, } \\
& \frac{d x_{e}(t)}{d t}=\left(\begin{array}{ll}
A & 0 \\
-K C & A-K C
\end{array}\right) x_{e}(t), \\
& e_{y}(t)=y(t)-C x_{o}(t) \text {, } \\
& x_{e t}(t)=\left(\begin{array}{cc}
x(t) & x(t)-x_{o}(t)
\end{array}\right)^{T}=L_{e} x_{e}(t), \\
& \frac{d x_{e t}(t)}{d t}=\left(\begin{array}{ll}
A & 0 \\
0 & A-K C
\end{array}\right) x_{e t}(t), \\
& e_{y}(t)=y(t)-C x_{o}(t)=\left(\begin{array}{ll}
0 & C
\end{array}\right) x_{e t}(t) .
\end{aligned}
$$

As is well known in control theory, if the tuple $(A, C)$ is an observable pair then there exists a gain matrix $K$ such that the observable part of the performance system is globally asymptotically stable. Note the nonobservability of the performance system with respect to $e_{y}$ !

Example VI.4 This is a continuation of Example IV.11 If $k_{1}<-a_{22}$ and if $k_{2}<0$ then the observable part of the performance system is locally stable at $x-x_{o}=0$. The system itself is such that its linearized system at $x=0$ has one eigenvalue at zero. The global asymptotic stability is not yet established. 


\section{EXAMPLES}

Example VII.1 A polynomial system with a polynomial observer of higher state-space dimension than the system. There follows an example of an observer for a system in which the state-space dimension of the observer is strictly larger than that of the system.

Consider the polynomial system and derive its observer according to the steps,

$$
\begin{aligned}
\frac{d x(t)}{d t}= & \left(\begin{array}{l}
f_{1}(x(t)) \\
-a_{21} x_{2}(t)+a_{22}
\end{array}\right), \\
& x(0)=x_{0}, \\
y(t)= & x_{1}(t)=C x(t)=\left(\begin{array}{cc}
1 & 0
\end{array}\right) x(t), \\
f_{1}(x)= & 2 a_{21} x_{1}-a_{12}\left(x_{2}-a_{13}\right)\left(x_{2}-a_{14}\right), \\
& a_{12}, a_{13}, a_{14}, a_{21}, a_{22} \in(0, \infty), \\
& a_{13}<\frac{a_{22}}{a_{21}}<a_{14}, a_{13}+a_{14} \neq 2 \frac{a_{22}}{a_{21}} . \\
s_{1}(x)= & h(x)=C x=x_{1}, \\
s_{2}(x)= & \frac{\partial h(x)}{\partial x} f(x)=f_{1}(x), \\
\hat{x}_{1}= & s_{1}(x)=x_{1}, x_{1}=\hat{x}_{1}, \\
\hat{x}_{2}= & s_{2}(x)=2 a_{21} x_{1}-a_{12}\left(x_{2}-a_{13}\right)\left(x_{2}-a_{14}\right) \\
= & 2 a_{21} \hat{x}_{1}-a_{12}\left(x_{2}-a_{13}\right)\left(x_{2}-a_{14}\right) .
\end{aligned}
$$

The conclusion is that based on the state-space transformation $s=\left(s_{1}, s_{2}\right)$ there does not exist an unique solution for the inverse function, in particular not a solution for $x_{2}$ given $\hat{x}_{1}, \hat{x}_{2}$ of the equation

$$
a_{12}\left(x_{2}-a_{13}\right)\left(x_{2}-a_{14}\right)=-\hat{x}_{2}+2 a_{21} \hat{x}_{1} .
$$

Therefore the construction of the state-space transformation is increased by another derivation.

$$
\begin{aligned}
s_{3}(x) & =\frac{\partial s_{2}(x)}{\partial x} f(x)=c_{11} x_{1}+c_{12} x_{2}+c_{14}, \\
c_{11} & =4 a_{21}^{2}, \\
c_{12} & =a_{12} a_{21}\left(a_{13}+a_{14}\right)-2 a_{12} a_{22}, \\
c_{14} & =a_{12} a_{22}\left(a_{13}+a_{14}\right)-2 a_{12} a_{21} a_{13} a_{14} . \\
\hat{x}_{3} & =s_{3}(x), \\
x_{2} & =-\frac{c_{11}}{c_{12}} \hat{x}_{1}+\frac{1}{c_{12}} \hat{x}_{3}-\frac{c_{14}}{c_{12}}, \\
\left(x_{1}, x_{2}\right) & =s^{-1}(\hat{x})=\left(\hat{x}_{1},-\frac{c_{11}}{c_{12}} \hat{x}_{1}+\frac{1}{c_{12}} \hat{x}_{3}-\frac{c_{14}}{c_{12}}\right), \\
\frac{d \hat{x}(t)}{d t} & =A_{\text {or }} \hat{x}(t)+b_{\text {or }}(\hat{x}(t)), \hat{x}(t), x_{o}(t) \in \mathbb{R}^{3} .
\end{aligned}
$$

Note that $s^{-1}$ is a linear map. Then,

$$
\begin{aligned}
s_{4}(x)= & 2 a_{21} c_{11} x_{1}-a_{21} c_{12} x_{2}+ \\
& -a_{12} c_{11}\left(x_{2}-a_{13}\right)\left(x_{2}-a_{14}\right)+a_{22} c_{12}, \\
b_{o r, 3}(\hat{x})= & \left.s_{4}(x)\right|_{x=s^{-1}(\hat{x})} \\
= & 3 a_{21} c_{11} \hat{x}_{1}-a_{21} \hat{x}_{3}+a_{21} c_{14}+a_{22} c_{12}+ \\
& -\frac{a_{12} c_{11}}{c_{12}^{2}} \times \\
& \times\left[\hat{x}_{3}-c_{11} \hat{x}_{1}-\left(c_{14}+a_{13} c_{12}\right)\right] \times \\
& \times\left[\hat{x}_{3}-c_{11} \hat{x}_{1}-\left(c_{14}+a_{14} c_{12}\right)\right],
\end{aligned}
$$

$$
\begin{aligned}
k_{o, 3}(\hat{x})= & \frac{\partial b_{o r, 3}(\hat{x})}{\partial \hat{x}_{1}}=3 a_{21} c_{11}+\frac{a_{12} c_{11}^{2}}{c_{12}^{2}} \times \\
& \times\left[\begin{array}{l}
+2 \hat{x}_{3}-2 c_{11} \hat{x}_{1}+ \\
-\left(2 c_{14}+c_{12}\left(a_{13}+a_{14}\right)\right)
\end{array}\right] .
\end{aligned}
$$

The observer is then described by the system representation,

$$
\begin{aligned}
\frac{d x_{o}(t)}{d t}= & A_{o} x_{o}(t)+b_{o}\left(x_{o}(t)\right)+ \\
& +\left[k_{o}\left(x_{o}(t)\right)+K\right]\left[y(t)-C_{o} x_{o}(t)\right] \\
= & \left(\begin{array}{l}
\hat{x}_{2}(t) \\
\hat{x}_{3}(t) \\
b_{o, 3}(\hat{x}(t))
\end{array}\right)+ \\
& +\left(\begin{array}{l}
k_{1} \\
k_{2} \\
k_{3}+k_{o, 3}(\hat{x}(t))
\end{array}\right)\left[y(t)-C_{o} \hat{x}(t)\right], \\
y_{o}(t)= & C_{o} x_{o}(t), \quad C_{o}=\left(\begin{array}{lll}
1 & 0 & 0
\end{array}\right) .
\end{aligned}
$$

Example VII.2 Rational system - rational observer. Consider the observable rational system, for $a_{13} \neq 0$.

$$
\begin{aligned}
\frac{d x(t)}{d t} & =\left(\begin{array}{l}
\frac{-a_{11} x_{1}(t)}{1+a_{12} x_{1}(t)}+\frac{a_{13} x_{2}(t)}{1+a_{14} x_{2}(t)} \\
\frac{-a_{21} x_{2}(t)}{1+a_{22} x_{2}(t)}+a_{23}
\end{array}\right), \\
y(t) & =C x(t)=\left(\begin{array}{ll}
1 & 0
\end{array}\right) x(t)=x_{1}(t) .
\end{aligned}
$$

Construct an output-based realization according to Procedure IV.1

$$
\begin{aligned}
\frac{d \hat{x}(t)}{d t}= & A_{\text {or }} \hat{x}(t)+b_{\text {or }}(\hat{x}(t)) \\
= & \left(\begin{array}{ll}
0 & 1 \\
0 & 0
\end{array}\right) \hat{x}(t)+\left(\begin{array}{l}
0 \\
b_{o r, 2}(\hat{x}(t))
\end{array}\right), \\
y(t)= & C \hat{x}(t), \\
b_{o r, 2}(x)= & \frac{a_{11}^{2} x_{1}}{\left(1+a_{12} x_{1}\right)^{2}}-\frac{a_{11} a_{13} x_{2}}{\left(1+a_{12} x_{1}\right)^{2}\left(1+a_{14} x_{2}\right)}+ \\
& -\frac{a_{13} a_{21} x_{2}}{\left(1+a_{14} x_{2}\right)^{2}\left(1+a_{22} x_{2}\right)}+\frac{a_{13} a_{23}}{\left(1+a_{14} x_{2}\right)^{2}} .
\end{aligned}
$$

The rational observer follows.

$$
\begin{aligned}
& \frac{d x_{o}(t)}{d t}= A_{o} x_{o}(t)+b_{o}\left(x_{o}(t)\right)+ \\
&+\left[k_{o}\left(x_{o}(t)\right)+K\right]\left[y(t)-C x_{o}(t)\right], \\
& A_{o}= A_{o r}, \\
& b_{o}\left(x_{o}\right)= b_{o r}\left(x_{o}\right)=\left(\begin{array}{l}
0 \\
b_{o r, 2}\left(x_{o}\right)
\end{array}\right), \\
& k_{o, 2}\left(x_{o}\right)=\frac{\partial b_{o r, 2}\left(x_{o}\right)}{\partial x_{o, 1}} \\
&=\frac{a_{11}^{2}}{\left(1+a_{12} x_{o, 1}\right)^{3}}-\frac{3 a_{11} a_{12} x_{o, 1}}{\left(1+a_{12} x_{o, 1}\right)^{4}}+ \\
&+\frac{2 a_{12}}{\left(1+a_{12} x_{o, 1}\right)^{3}} \frac{a_{11} a_{13} x_{o, 2}}{\left(1+a_{14} x_{o, 2}\right)}+ \\
&-\frac{a_{13} a_{14} x_{o, 2}}{\left(1+a_{14} x_{o, 2}\right)^{2}\left(1+a_{22} x_{o, 2}\right)} .
\end{aligned}
$$

Example VII.3 Consider a rational system which is a model for either a compartmental system with two compartments or a biochemical reaction system with two chemical species. 
The output is only of the second compartment or species. The system is actually a family of rational systems.

$$
\begin{aligned}
\frac{d x_{1}(t)}{d t} & =r_{1}\left(x_{1}(t)\right)-r_{2}\left(x_{1}(t), x_{2}(t)\right), x_{1}(0)=x_{1,0}, \\
\frac{d x_{2}(t)}{d t} & =r_{2}\left(x_{1}(t), x_{2}(t)\right)-r_{3}\left(x_{2}(t)\right), x_{2}(0)=x_{2,0}, \\
y(t) & =C x(t)=x_{2}(t), r_{1}, r_{2}, r_{3} \in Q_{X} .
\end{aligned}
$$

Assume that $r_{2}\left(0, x_{2}\right)=0$ and $r_{3}(0)=0$ for all $x_{2} \in[0, \infty)$ and that $x_{0}=\left(x_{0,1}, x_{0,2}\right) \in \mathbb{R}_{+}^{2}$. From those assumptions follows that the positive orthant $\mathbb{R}_{+}^{2}=[0, \infty)^{2}$ is an invariant set to be denoted as the state set $X=\mathbb{R}_{+}^{2}$. A special case of the above is with

$$
\begin{aligned}
& r_{1}\left(x_{1}\right)=a_{1}, r_{2}\left(x_{1}, x_{2}\right)=\frac{a_{2} x_{1} x_{2}}{1+a_{3} x_{2}^{2}}, \\
& r_{3}\left(x_{2}\right)=a_{4} x_{2}, \quad a_{1}, a_{2}, a_{3}, a_{4} \in(0, \infty) .
\end{aligned}
$$

The state-space transformation and its inverse are then

$$
\begin{aligned}
& \hat{x}_{1}=s_{1}(x)=h(x)=C x=x_{2}, \\
& \hat{x}_{2}=s_{2}(x)=\frac{\partial s_{1}(x)}{\partial x}=f_{2}(x)=r_{2}\left(x_{1}, x_{2}\right)-r_{3}(x), \\
& x_{2}=\hat{x}_{1}, \quad x_{1}=\frac{\left(\hat{x}_{2}-a_{4} \hat{x}_{1}\right)\left(1+a_{3} \hat{x}_{1}^{2}\right)}{a_{2} \hat{x}_{1}} .
\end{aligned}
$$

The output-based realization is then,

$$
\begin{aligned}
\frac{d \hat{x}(t)}{d t}= & \left(\begin{array}{l}
\hat{x}_{2}(t) \\
b_{2}(\hat{x}(t))
\end{array}\right), \hat{y}(t)=C \hat{x}(t), \\
b_{o, 2}(\hat{x})= & p_{o, 2}\left(x_{o}\right) /\left[a_{2} x_{o, 1}\left(1+a_{3} x_{o, 1}^{2}\right)\right], \\
p_{o, 2}\left(x_{o}\right)= & -2 a_{2} x_{o, 1}^{2}+a_{4}^{2} x_{o, 1}\left(1+a_{3} x_{o, 1}^{2}\right)^{2}+ \\
& +\left(1+a_{3} x_{o, 1}^{2}\right)\left[a_{2} x_{o, 1}\left(a_{1}-2 a_{4} x_{o, 1}\right)+\right. \\
& +2\left(x_{o, 2}+a_{4} x_{o, 1}\right)+ \\
& \left.-2 a_{1} x_{o, 1}^{4}\left(x_{o, 2}+a_{4} x_{o, 1}\right)^{2}\right] .
\end{aligned}
$$

The observer is then,

$$
\begin{aligned}
\frac{d x_{o}(t)}{d t}= & \left(\begin{array}{l}
x_{o, 2}(t) \\
b_{o}\left(x_{o}(t)\right)
\end{array}\right)+ \\
& +\left[k_{o}\left(x_{o}(t)\right)+K\right]\left(y(t)-C_{o} x_{o}(t)\right) .
\end{aligned}
$$

\section{CONCLUDING REMARKS}

The main contribution of the paper is an observer synthesis procedure for rational observers of rational systems.

Further research into the subject of this paper includes: Synthesis of observers for rational systems with inputs (first for systems affine in the input). The role of universal inputs has to be explored. A stability analysis of rational observers. Observers for Nash systems [3] have been constructed but are not discussed in this paper due to space limitations.

\section{REFERENCES}

[1] J. Němcová and J. H. van Schuppen, "Realization theory for rational systems: The existence of rational realizations," SIAM J. Control \& Opt., vol. 48, pp. 2840 - 2856, 2009.

[2] J. Němcová and J. van Schuppen, "Realization theory for rational systems: Minimal realizations," Acta Applicandae Mathematicae, vol. 110, pp. 605-626, 2010.

[3] J. Němcová, M. Petreczky, and J. H. van Schuppen, "Realization theory of Nash systems," SIAM J. Control \& Opt., vol. 51, pp. 33863414, 2013.
[4] R. Kalman, "New methods in Wiener filtering theory," in Proceedings 1st Symposium Engineering Applications of Random Function Theory and Probability, J. Bogdanoff and F. Kozin, Eds. New York: Wiley, 1963, pp. 270-388.

[5] Y. Wang, J. H. van Schuppen, and J. Vrancken, "On-line distributed prediction of traffic flow in a large-scale road network," Simulation Modelling Practice and Theory, vol. 47, pp. 276-303, 2014.

[6] J. Huang, Nonlinear Output Regulation: Theory and Applications, ser. Advances in Design and Control. Philadelphia: SIAM, 2004, seen 2005:01:20, very good, no other book on this specific topic.

[7] J. Němcová, M. Petreczky, and J. H. van Schuppen, "An algorithm for system identification of a discrete-time polynomial system without inputs," in Proc. IFAC Symposium System Identification (SYSID.2015), E.-W. Bai and J. Zhang, Eds., IFAC. Laxenburg: IFAC, 2015, pp. 166-171.

[8] R. Kalman, "A new approach to linear filtering and prediction problems," J. Basic Eng., vol. 82, pp. 35-45, 1960.

[9] D. Luenberger, "Observing the state of a linear system," IEEE Trans. Military Electronics, vol. 23, pp. 119-125, 1964.

[10] — - "Observers for multivariable systems," IEEE Trans. Automatic Control, vol. 11, pp. 190-197, 1966.

[11] A. Isidori, Nonlinear control systems (3rd. ed.). Berlin: Springer, 2001.

[12] G. Besancon, Ed., Nonlinear observers and applications. Berlin: Springer, 2007.

[13] H. Nijmijer and T. Fossen, Eds., New directions in nonlinear observer design, ser. Lecture Notes in Control and Information Sciences. Berlin: Springer, 1999, no. 244.

[14] J. Gauthier and I. Kupke, "Observability and observers for nonlinear systems," SIAM J. Control \& Opt., vol. 32, pp. 975-994, 1994.

[15] — Deterministic observation theory and applications. Cambridge: Cambridge University Press, 2001

[16] M. Chaves and E. Sontag, "Observers for chemical reaction networks," in Proceedings European Control Conference 2001, Porto, Portugal, 2001, pp. 3715-3720.

[17] E. Sontag, Polynomial response maps, ser. Lecture Notes in Control and Information Sciences. Berlin: Springer-Verlag, 1979, vol. 13.

[18] Z. Bartosiewicz, "Minimal polynomial realizations," Math. Control Signals Systems, vol. 1, pp. 227 - 237, 1988.

[19] B. Jakubczyk, "Existence and uniqueness of realizations of nonlinear systems," SIAM J. Control \& Opt., vol. 18, pp. 455-471, 1980.

[20] Y. Wang and E. D. Sontag, "Algebraic differential equations and rational control systems," SIAM J. Control \& Opt., vol. 30, pp. 11261149,1992

[21] O. Zariski and P. Samuel, Commutative algebra - Volume 1. New York: Springer, 1958

[22] D. Cox, J. Little, and D. O'Shea, Ideals, varieties, algorithms: An introduction to computational algebraic geometry and commutative algebra, ser. Undergraduate Texts in Mathematics. Berlin: Springer, 1992.

[23] E. Kunz, Introduction to commutative algebra and algebraic geometry. Stuttgart: Birkhäuser, 1985

[24] J. Bochnak, M. Coste, and M.-F. Roy, Real algebraic geometry, ser. Ergebnisse der Mathematik und ihrer Grenzgebiete. Berlin: Springer, 1998, no. 39.

[25] E. Sontag, Mathematical control theory: Deterministic finite dimensional systems (2nd. Ed.), ser. Graduate Text in Applied Mathematics. New York: Springer, 1998, no. 6.

[26] J. Ortega and W. Rheinboldt, Iterative solutions of nonlinear equations in several variables. New York: Academic Press, 1970.

[27] T. Kailath, Linear systems. Englewood Cliffs: Prentice-Hall Inc., 1980

[28] K. Murota, Systems analysis by graphs and matroids - Structural solvability and controllability, ser. Algorithms and combinatorics. Berlin: Springer-Verlag, 1987, vol. 3.

[29] A. van den Essen, Polynomial automorphisms - and the Jacobi conjecture. Basel: Birkhäuser, 2000.

[30] — "A criterion to decide if a polynomial map is invertible and to compute the inverse," Comm. in Algebra, vol. 10, pp. 3183-3186, 1990.

[31] A. Vannelli and M. Vidyasagar, "Maximal lyapunov functions and domains of attraction for autonomous nonlinear systems," Automatica, vol. 21, pp. 69-80, 1985. 